\title{
Pacific
}

Journal of

Mathematics

\section{PERIODICITY, GENERA AND ALEXANDER POLYNOMIALS} OF KNOTS

SWATEE NAIK

Volume $166 \quad$ No. 2

December 1994 


\title{
PERIODICITY, GENERA AND ALEXANDER POLYNOMIALS OF KNOTS
}

\author{
SWATEE NAIK
}

For knots in $S^{3}$ criteria for periodicity are obtained in terms of the homology groups of cyclic branched covers of $S^{3}$ and the Alexander polynomial. Also the relationship between the genus of a periodic knot and the Alexander polynomial is studied. As an application it is shown that no eleven crossing knot has a period greater than 5 .

1. Introduction. A knot $K$ in $S^{3}$ is said to be periodic if there exists an integer $q>1$ and an orientation preserving diffeomorphism $f: S^{3} \rightarrow S^{3}$ such that $f(K)=K$, order $(f)=q$, and the fixed point set of $f$ is a circle disjoint from $K$. Any such $q$ is called a period of $K$, and any such $f$, a corresponding periodic transformation.

A natural question is how to determine whether a knot is periodic with a given period. R. Fox [8] conjectured in 1962 that a nontrivial knot has only finitely many periods. This was first proved by E. Flapan [7] in 1983 and an explicit bound for the possible periods of a knot, in terms of its genus, was given by A. Edmonds [6] in 1984.

In 1971, K. Murasugi [13] showed that the Alexander polynomial of a period $q$ knot has to satisfy certain conditions. Several other techniques to determine the possible periods for a given knot have been developed since then. The more recent results include criteria involving other polynomial invariants (see $[14,16,18,19,20,21])$ and hyperbolic structures on knot complements [1]. The efficacy of the Murasugi conditions lies in the simplicity of these conditions, the computability of the Alexander polynomial, and the fact that an Alexander polynomial occurs as the polynomial of an infinite collection of knots, thereby making the results applicable to a large class. 
What makes the Alexander polynomial results even more interesting, is the recent "converse" obtained by J. Davis and C. Livingston [5]. They show that with some additional restrictions, the Murasugi conditions on a polynomial guarantee the existence of a periodic knot with that as its Alexander polynomial.

In this paper we effectively utilize the lifting of the periodic action to the finite cyclic covers of $S^{3}$ branched over a periodic knot to obtain conditions on the homology groups of these covers. Our results provide simple and useful criteria for periodicity of a knot. More important, they bring out an interplay between the genus of a periodic knot and the Alexander polynomial. We show that a purely algebraic hypothesis on the polynomial gives us fairly large lower bounds on the genus of any periodic knot with that as its Alexander polynomial. As a quick example, consider the polynomial $7 t^{6}-13 t^{3}+7$. By [5], it follows that this is the polynomial of a period 7 knot. Our results show that any period 7 knot with this polynomial has genus at least as big as 21 , and this will be seen to imply that such a knot has at least 44 crossings.

We also observe that results of [6] can be sharpened using the Murasugi conditions and these new results give powerful tools to determine the possible periods higher than the genus of the knot. As an application, we can easily show that out of the 552 knots with 11 crossings, only 3 may have period 5, and no 11-crossing knot has a period greater than 5 . These remaining difficult cases of possible period 5 can be ruled out with more subtle techniques.

The paper is organized as follows.

In Section 2 we obtain conditions on the torsion submodules of the homology modules of the finite cyclic covers of $S^{3}$ branched over a periodic knot and also give criteria for periodicity in terms of the Alexander polynomial. In particular, for any prime $l$ such that there is no $l$-torsion in the homology of the cyclic cover of $S^{3}$ branched over the quotient knot, Theorem 2.6 gives an explicit characterization of the $l$-Sylow subgroup of the torsion group of the periodic knot. The result of 2.6 is shown to be independent of the Murasugi conditions through the following example.

The Alexander polynomial of the knot $10_{3}$ satisfies the Murasugi conditions for period 3 (see 4.1). The first homology group of the 2-fold cyclic cover of $S^{3}$ branched over $10_{3}$ is the cyclic group of 
order 25. We will show that this contradicts 2.6 , thereby proving that $10_{3}$ does not have period 3 . Note that this was shown before in [18] using the skein polynomial.

In Section 3 we discuss the genus of a periodic knot with a given Alexander polynomial.

In Section 4 we combine results of [6] with the Murasugi conditions to obtain better criteria for periods higher than the genus of a knot.

Finally, in Section 5, we consider various examples of 10- and 11- crossing knots, where by applying the results of the previous sections, we give a short proof of previously known results in the case of 10 - crossing knots and prove new results for 11- crossing knots.

Thanks are due to James F. Davis, Sudhir R. Ghorpade, and Charles Livingston for all their help.

2. Cyclic Covers of $S^{3}$ branched over a periodic knot. Let $K \subset S^{3}$ be a periodic knot with a period $q$ and $f: S^{3} \rightarrow S^{3}$ be the corresponding periodic transformation. We have $f^{q}=\operatorname{id}_{S^{3}} \neq$ $f^{n}$, for $1 \leq n<q$. Let $\left(M_{k}, \theta\right)$ be the $k$-fold cyclic cover of $S^{3}$ branched over $K$. We will first show that the map $f$ can be lifted to an order $q$ homeomorphism of $M_{k}$ onto itself. To this end, let us briefly recall the construction of $M_{k}$.

Take a tubular neighborhood $T$ of $K$ in $S^{3}$ such that $f(T)=T$. The existence of such a neighborhood $T$ follows from the Equivariant Tubular Neighborhood Theorem (see Chapter VI, Theorem 2.2 of [2]). Let $X=S^{3}-\operatorname{int}(T)$, and let $\left(X_{k}, \theta\right)$ be the $k$-fold cyclic cover of $X$. The boundary $\partial X_{k}$ is a torus that covers $\partial X$ by wrapping the meridian of $\partial X_{k}$ around the meridian of $T, k$-times. The branched cover $M_{k}$ is obtained by attaching a solid torus $D^{2} \times S^{1}$ to $X_{k}$, along $\partial X_{k}$, in such a way that $\partial D^{2} \times\{\mathrm{pt}\}$ is identified with the meridian of $\partial X_{k}$. The covering projection $\theta: X_{k} \rightarrow X$ clearly extends to the desired branched covering $M_{k} \rightarrow S^{3}$, which we may also denote by $\theta$. In the following lemma we show that $\left.f\right|_{X}$ can be lifted to an order $q$ homeomorphism of the unbranched cover $X_{k}$.

LEMMA 2.1. There exists an order $q$ homeomorphism $\tilde{f}: X_{k} \rightarrow$ 
$X_{k}$ such that $\theta \circ \tilde{f}=f \circ \theta$.

Proof. Let the restriction of $f$ to $X$ also be denoted by $f$. Choose a base point $s$ for $\pi_{1}(X)$ in the fixed point set of $f$, and a base point $\tilde{s}$ for $\pi_{1}\left(X_{k}\right)$ in $\theta^{-1}(s)$. Note that $\pi_{1}(\theta)\left(\pi_{1}\left(X_{k}\right)\right)$ is the only normal subgroup of $\pi_{1}(X)$ of index $k$ with a cyclic quotient. It follows that it is a characteristic subgroup. Now, since $f$ is a homeomorphism, $\pi_{1}(f)$ is an automorphism of $\pi_{1}(X)$, and thus

$$
\pi_{1}(f) \circ \pi_{1}(\theta)\left(\pi_{1}\left(X_{k}\right)\right)=\pi_{1}(\theta)\left(\pi_{1}\left(X_{k}\right)\right) .
$$

Consequently, there exists a map $\tilde{f}: X_{k} \rightarrow X_{k}$, which fixes $\tilde{s}$ and lifts $f \circ \theta$, i.e., $\theta \circ \tilde{f}=f \circ \theta$. By induction, $\theta \circ(\tilde{f})^{i}=f^{i} \circ \theta$, for $i \geq 1$. Since $f$ has order $q,(\tilde{f})^{i} \neq \operatorname{id}_{X_{k}}$ for $1 \leq i<q$ and $\theta \circ(\tilde{f})^{q}=\theta$. Thus $(\tilde{f})^{q}$ is a covering transformation, which fixes the point $\tilde{s}$, and therefore $(\tilde{f})^{q}=\operatorname{id}_{X_{k}}$. It follows that $\tilde{f}$ is an order $q$ homeomorphism.

It is easy to see that $\left.\tilde{f}\right|_{\partial X_{k}}$ can be extended to an order $q$ selfhomeomorphism of $D^{2} \times S^{1}$. As a consequence, we have the following.

Proposition 2.2. There exists an order $q$ homeomorphism $\hat{f}$ : $M_{k} \rightarrow M_{k}$ such that $\theta \circ \hat{f}=f \circ \theta$.

REMARK 2.3. It can easily be seen that the lifting $\hat{f}$ is, in fact, a diffeomorphism with respect to the induced differentiable structure on the covering space.

In the remainder of this section, we obtain necessary conditions which the homology modules of the branched covers, and consequently the Alexander polynomial, must satisfy in order that the corresponding knot is periodic with a period $q$. Note that the map $\hat{f}$ defines an action of $C_{q}$, the cyclic group of $q$ elements, on $M_{k}$. This action induces an action of the group ring $Z\left[C_{q}\right]$ on $H_{1}\left(M_{k}\right)$. Let $l$ be a prime and let $H_{1}\left(M_{k}\right)_{l}$ [resp: $H_{1}\left(M_{k} / C_{q}\right)_{l}$ ] denote the subgroup of $H_{1}\left(M_{k}\right)$ [resp: $H_{1}\left(M_{k} / C_{q}\right)$ ] consisting of the elements of order a power of $l$. Clearly, $Z\left[C_{q}\right]$ acts on $H_{1}\left(M_{k}\right)_{l}$. Let $\left(H_{1}\left(M_{k}\right)_{l}\right)^{C_{q}}$ denote the set of fixed points of the action.

Proposition 2.4. If $q$ is a prime, then $H_{1}\left(M_{k}\right)_{l} /\left(H_{1}\left(M_{k}\right)_{l}\right)^{C_{q}}$ 
is a $Z\left[\zeta_{q}\right]$-module. Here $\zeta_{q}$ is a primitive $q$-th root of unity.

Proof. Let $t$ be a generator of $C_{q}$ and let $\sigma$ be the element $1+$ $t+\cdots+t^{q-1}$ of $Z\left[C_{q}\right]$. Since $q$ is a prime, $Z\left[C_{q}\right] /\langle\sigma\rangle \cong Z\left[\zeta_{q}\right]$. Also, $\sigma \alpha \in\left(H_{1}\left(M_{k}\right)_{l}\right)^{C_{q}}$ for all $\alpha \in H_{1}\left(M_{k}\right)_{l}$. The result follows.

PROPOSITION 2.5. If the prime $l$ is relatively prime to $q$, then

$$
\left(H_{1}\left(M_{k}\right)_{l}\right)^{C_{q}} \cong H_{1}\left(M_{k} / C_{q}\right)_{l} \text {. }
$$

Proof. Let $\pi_{*}: H_{1}\left(M_{k}\right)_{l}^{C_{q}} \rightarrow H_{1}\left(M_{k} / C_{q}\right)_{l}$ be the restriction of the map induced by the quotient $M_{k} \rightarrow M_{k} / C_{q}$, and let $\mu_{*}: H_{1}\left(M_{k} / C_{q}\right)_{l} \rightarrow H_{1}\left(M_{k}\right)_{l}^{C_{q}}$ be the transfer map. It is easy to see that the composite maps $\pi_{*} \circ \mu_{*}$ and $\mu_{*} \circ \pi_{*}$ are multiplications by $q$ on the respective modules. Since $q$ and $l$ are relatively prime, we have the result. (Compare the proof of Theorem 2.4, Chapter III of [2].)

Notation. For distinct primes $l$ and $q$, let $f_{q}(l)$ denote the multiplicative order of $l(\bmod q)$, i.e., the least positive integer such that $l^{f_{q}(l)} \equiv 1(\bmod q)$.

THEOREM 2.6. Let $K$ be a periodic knot with a prime period $q$ and let $H_{1}\left(M_{k} / C_{q}\right)_{l}=0$ for some prime $l \neq q$. Then there exist nonnegative integers $t, a_{1}, \cdots, a_{t}$ such that

$$
H_{1}\left(M_{k}\right)_{l} \cong\left(C_{l}\right)^{a_{1} f_{q}(l)} \oplus\left(C_{l^{2}}\right)^{a_{2} f_{q}(l)} \oplus \cdots \oplus\left(C_{l^{t}}\right)^{a_{t} f_{q}(l)} .
$$

Proof. From 2.4 and 2.5 we see that $H_{1}\left(M_{k}\right)_{l}$ is a $Z\left[\zeta_{q}\right]$-module. We have

$$
H_{1}\left(M_{k}\right)_{l} \cong \Lambda / L_{1}^{n_{1}} \oplus \cdots \oplus \Lambda / L_{s}^{n_{s}},
$$

where $\Lambda=Z\left[\zeta_{q}\right], L_{1}, \cdots, L_{s}$ are prime ideals of $\Lambda$, and $n_{1}, \cdots, n_{s}$ are positive integers (see [10]). Using standard results from Algebraic Number Theory it can be seen that $l$ is unramified in $\Lambda$, and if $L$ is a prime ideal in $\Lambda$ such that $L \cap Z=l Z$, then

$$
\Lambda / L^{n} \cong{ }_{Z}\left(C_{l^{n}}\right)^{f_{q}(l)} .
$$


This gives the desired isomorphism. (Also see the characterization of $Z\left[\zeta_{q}\right]$-modules, as given in Theorem 2.5 of [4]. It may be noted that this result in [4] is misstated. In the notation of (2.5) of [4], $G_{l}$ should be defined as the set of elements of $G$ of order a power of $l$ instead of those of order divisible by $l$; with this modification the proof given in [4] is correct.)

It is easy to see that $M_{k} / C_{q}$ is the $k$-fold cyclic cover of $S^{3}$ branched over the quotient knot $K / C_{q}$. This makes the above theorem more easily applicable to examples as illustrated below.

ExAmple. Consider the knot $10_{3}$ listed as having a possible period 3 in [3]. We have, $H_{1}\left(M_{2}\right)=C_{25}$, and if this knot had period 3 , the quotient knot would be trivial (to prove this, use Theorem 3.2 of the next section along with the fact that $10_{3}$ has genus 1 ). Thus, $H_{1}\left(M_{2} / C_{3}\right)$ would be trivial, and since $f_{3}(5)=2$, Theorem 2.6 is contradicted. Thus $10_{3}$ does not have period 3. (See also [18].)

In 2.7 below, we obtain a quick corollary to Theorem 2.6. For a finitely generated abelian group $G$, let $|G|$ denote the order of its torsion subgroup.

CoROllary 2.7. Let $K$ be a periodic knot with a prime period $q$ and let $\bar{K}$ be the image of $K$ in $S^{3} / C_{q}$. Let $M_{k}$ and $\bar{M}_{k}$ be the $k$ fold cyclic covers of $S^{3}$ branched over $K$ and $\bar{K}$, respectively. Then for each prime factor $l \neq q$ of $\left|H_{1}\left(M_{k}\right)\right|$, which does not divide $\left|H_{1}\left(\bar{M}_{k}\right)\right|$, we have $l^{f_{q}(l)}|| H_{1}\left(M_{k}\right) \mid$.

Note that (see Section 5 of [9]), if $\zeta_{k}$ is a primitive $k$-th root of unity, we have

$$
\left|H_{1}\left(M_{k}\right)\right|=\left|\prod_{i=1}^{k} \Delta\left(\zeta_{k}^{i}\right)\right| \text { and }\left|H_{1}\left(\bar{M}_{k}\right)\right|=\left|\prod_{i=1}^{k} \bar{\Delta}\left(\zeta_{k}^{i}\right)\right| .
$$

Using the factorization of Alexander polynomials of periodic knots obtained in [13], we can, in fact, prove a stronger statement than 2.7. Note that the Alexander polynomial is well defined only up to multiplication by units in $\mathbf{Z}\left[t, t^{-1}\right]$; we normalize it so that it has a nonzero constant term and it contains no negative powers of $t$.

THEOREM 2.8. Let $K$ be a periodic knot with a prime period $q$, let $\Delta$ and $\bar{\Delta}$ be the Alexander polynomials of $K$ and of its quotient 
$k n o t$, respectively. Let $l$ be a prime such that $l \neq q$, let $f_{q}(l)$ denote the multiplicative order of $l$ mod $q$, and for a positive integer $m$, let $\zeta_{m}$ denote a primitive $m$-th root of unity. Then the following statements are true.

(i) If $a \in Z$ and $l \mid(\Delta / \bar{\Delta})(a)$, then $l^{f_{q}(l)} \mid(\Delta / \bar{\Delta})(a)$.

(ii) If $k \geq 2$ and $l \mid \prod_{i=1}^{k}(\Delta / \bar{\Delta})\left(\zeta_{k}^{i}\right)$, then $l^{f_{q}(l)} \mid \prod_{i=1}^{k}(\Delta / \bar{\Delta})\left(\zeta_{k}^{i}\right)$.

Proof. It was shown in [13] (see also (14.21) of [3]) that $\bar{\Delta}$ divides $\Delta$ in $\mathbf{Z}[t]$ and $\Delta / \bar{\Delta}=\prod_{i=1}^{q-1} D\left(t, \zeta_{q}^{i}\right)$, where $D$ is a polynomial in 2 variables with integer coefficients. Note that $D\left(a, \zeta_{q}^{i}\right)$ and $\prod_{j=1}^{k-1} D\left(\zeta_{k}^{j}, \zeta_{q}^{i}\right)$ are in $\mathbf{Z}\left[\zeta_{q}\right]$, for $a \in Z, i \geq 1, k \geq 2$. Now, since $l \neq q$, we have, $l Z\left[\zeta_{q}\right]=L_{1} L_{2} \cdots L_{g}$, where $L_{1}, L_{2}, \cdots, L_{g}$ are distinct prime ideals of $\mathbf{Z}\left[\zeta_{q}\right]$ which are permuted transitively by $\operatorname{Gal}\left(Q\left(\zeta_{q}\right) / Q\right.$ ), and $g=(q-1) / f_{q}(l)$ (see Theorems 23 and 26 of $[\mathbf{1 2}])$. The result follows.

EXAMPLE. For the knot $11_{432}$ of $[\mathbf{1 5}], \Delta=\left(1+t-3 t^{2}+t^{3}+t^{4}\right)(1-$ $\left.t+t^{2}\right)^{2}$, and it follows from the Murasugi conditions that if it had period 3 , then $\bar{\Delta}=1-t+t^{2}$ (see 4.1). In that case $(\Delta / \bar{\Delta})(2)=5 \times 9$ and since $f_{3}(5)>1$, Theorem 2.8 ,(i) is contradicted. It follows that $11_{432}$ does not have period 3 .

3. The genus of periodic knots with a prescribed Alexander polynomial. Let us call a polynomial $\Delta \in Z[t]$ a knot polynomial if $\Delta(1)= \pm 1$ and $\Delta(t)=t^{\operatorname{deg} \Delta} \Delta\left(t^{-1}\right)$. A classical result of H. Seifert [17] states that for any knot polynomial $\Delta$, there exists a knot $K$ with $g(K)=\frac{1}{2} \operatorname{deg} \Delta$ and with $\Delta_{K}$, the Alexander polynomial of $K$, equal to $\Delta$. Note that the genus $g(K)$ of a knot $K$ is, by definition, the minimum of the genera of Seifert surfaces bounded by $K$. Recently, J. Davis and C. Livingston showed that if a knot polynomial $\Delta$ satisfies certain conditions, then there exists a periodic knot $K$ with $\Delta_{K}=\Delta$; as a particular case of their results, we have Theorem 3.1.

Notation. For polynomials $f$ and $g$ in $\mathbf{Z}[t]$, by $f \doteq g(\bmod q)$ we mean

$$
f \equiv \pm t^{n} g(\bmod q), \text { for some } n \in Z \text {. }
$$


THEOREM 3.1. (Corollary 1.2 of [5]). If $\Delta$ is a knot polynomial such that $\Delta(t) \doteq 1(\bmod q)$ for some integer $q>1$, then there exists a period $q$ knot with $\Delta$ as its Alexander polynomial.

Using the results of the previous section, we now show that for certain knot polynomials $\Delta$ with $\Delta \doteq 1(\bmod q)$ for some $q>1$, the genus of any period $q$ knot $K$ with $\Delta_{K}=\Delta$ has to be fairly high. We need the following result which is an immediate consequence of the last equation in the proof of Corollary 5 of [6], and may be thought of as the Riemann-Hurwitz Formula for periodic knots.

THEOREM 3.2. Let $K$ be a period $q$ knot with genus $g$ and let $\bar{g}$ be the genus of the quotient knot. Let $m$ be the number of points of intersection of the fixed set of the $C_{q}$ action and an equivariant Seifert surface $F$ of genus $g$ bounded by $K$. Then $g=q \cdot g\left(F / C_{q}\right)+$ $(q-1)(m-1) / 2$.

In particular, $g \geq q \bar{g}$.

THEOREM 3.3. Let $\Delta$ be a nontrivial knot polynomial, and let $q$ be a prime. Suppose that for each knot polynomial $f$ different from $\Delta$ and dividing $\Delta$, there exists an integer $a=a(f)$ and a prime $l=l(f)$ satisfying the following three conditions.
(i) $l \neq q$,
(ii) $l \mid(\Delta / f)(a)$
(iii) $l^{f_{q}(l)} \chi(\Delta / f)(a)$.

Then for any period $q$ knot $K$ with $\Delta_{K}=\Delta$, we have: $g(K) \geq$ $\frac{1}{2} q \operatorname{deg} \Delta$.

Proof. Let $K$ be any period $q$ knot with $\Delta_{K}=\Delta$. Let $\bar{K}=K / C_{q}$ and $\bar{\Delta}$ be the Alexander polynomial of $\bar{K}$. If $\bar{\Delta} \neq \Delta$, then by applying 2.8 with $a=a(\bar{\Delta})$ and $l=l(\bar{\Delta})$, we see that $l^{f_{q}(l)} \mid \Delta(a)$. But this contradicts our hypothesis. Now, by $3.2, g(K) \geq q g(\bar{K})$, and since $g(\bar{K}) \geq \frac{1}{2} \operatorname{deg} \bar{\Delta}$, we get the desired inequality.

ExAmple 1. Let $\Delta=7 t^{6}-13 t^{3}+7$. Clearly $\Delta$ is the Alexander polynomial of some knot. It is easy to see that $\Delta$ does not have any proper factors which are knot polynomials, and $\Delta(-1)=27$. Since $f_{7}(3)=6$, the hypothesis of 3.3 is clearly satisfied if we take $q=7$, $a=-1$, and $l=3$. It follows that the genus of any period $7 \mathrm{knot}$ with $\Delta$ as its Alexander polynomial, is at least 21 . 
EXAMPLE 2. The knot $10_{162}$ has the Alexander polynomial $\Delta=$ $3 t^{4}-9 t^{3}+11 t^{2}-9 t+3$. By 3.1 , there exists a period 3 knot with this Alexander polynomial. It is easy to see that $\Delta$ is an irreducible polynomial. Moreover, $\Delta(-1)=35$, which is divisible by 5 , and not by $5^{f_{3}(5)}=25$. Thus by 3.3 , we see that any period 3 knot with $\Delta$ as its Alexander polynomial must have genus $\geq 6$. Now the genus of a 10 -crossing knot is at most 4 (this follows from 4.5 in the following section). So $10_{162}$ cannot have period 3 . Note that this has been shown before by using hyperbolic structures on knot complements (see $[\mathbf{1}])$.

EXAMPLE 3. One can easily give examples of infinite families of "periodic polynomials" where the genera of the corresponding periodic knots are necessarily quite high. Let, for instance, $n$ and $k$ be powers of 3 and let $\Delta=\left(5 t^{2 n}-11 t^{n}+5\right)\left(10 t^{2 k}-19 t^{k}+\right.$ 10). By 3.1 , there exists a period 5 knot with $\Delta$ as its Alexander polynomial. Note that $f=5 t^{2 n}-11 t^{n}+5$ and $g=10 t^{2 k}-19 t^{k}+10$ are irreducible in $\mathbf{Z}[t]$ (apply Eisenstein's criterion to $f(t-1)$ and $g(t-1)$, respectively). By letting $a=-1, l(1)=l(f)=13$ and $l(g)=7$, and applying 3.3 , we see that the genus of any period 5 knot with this Alexander polynomial is at least $5(n+k)$.

4. Periods higher than the genus of a knot. Let $K$ be a nontrivial periodic knot with a period $q$ and $\bar{K}=K / C_{q}$ be the quotient knot. Let $B$ be the fixed set of the $C_{q}$ action. Let $\Delta$ and $g$ (resp: $\bar{\Delta}$ and $\bar{g}$ ) be the Alexander polynomial and the genus of $K$ (resp: $\bar{K}$ ). In this section we obtain some simple criteria for periods greater than the genus of the knot.

For convenience, let us restate some known results in 4.1 and 4.2 below, the proofs of which can be found in [13] and [6], respectively.

Theorem 4.1 Murasugi Conditions.

(4.1.1) $\bar{\Delta}$ divides $\Delta$.

(4.1.2) Let $q$ be a power of a prime $p$, and let $\lambda$ be the linking number $l k(B, K)$ of $B$ and $K$. Then

$$
\Delta \doteq(\bar{\Delta})^{q}\left(1+t+\cdots+t^{\lambda-1}\right)^{q-1}(\bmod p) \text {. }
$$

Moreover, $\lambda$ is relatively prime to $q$.

ThEOREM 4.2 EDMONDS' TheOREM. 
(4.2.1) $q \leq 2 g+1$.

(4.2.2) If $q>g$ and $m$ is the number of points of intersection of $B$ and an equivariant Seifert surface $F$ of genus $g$ bounded by $K$, then exactly one of the following possibilities holds:

(i) $q=g+1, \bar{g}=0$, and $m=3$.

(ii) $q=2 g+1, \bar{g}=0$, and $m=2$.

Both the above results have been very useful in determining the possible periods of a knot. Surprisingly, except in a few examples in Chapter 7 of [11], no attempt has been made so far of applying these together. When combined, these results give highly efficient criteria for periods higher than the genus of the knot, as observed in 4.3 below.

Proposition 4.3. Suppose $q$ is a power of a prime $p$, and $q>g$. Then $\bar{g}=0$ and exactly one of the following holds:

(i) $q=g+1, \lambda=1$, and $\Delta \doteq 1(\bmod p)$.

(ii) $q=g+1, \lambda=3, \operatorname{deg} \Delta=2(q-1)$, and $\Delta \doteq(1+t+$ $\left.t^{2}\right)^{q-1}(\bmod p)$.

(iii) $q=2 g+1, \lambda=2, \operatorname{deg} \Delta=q-1$, and $\Delta \doteq(1+t)^{q-1}(\bmod p)$.

Proof. Let $\lambda$ be as in 4.1 and $F$ be as in 4.2 . Let $P_{1}, \cdots, P_{m}$ be the points of intersection of $B$ and $F$. Note that $\lambda$ equals $\epsilon_{1}+\cdots+\epsilon_{m}$, for some $\epsilon_{1}, \cdots, \epsilon_{m} \in\{1,-1\}$. It follows that $\lambda \leq m$ and $\lambda \equiv m$ $(\bmod 2)$. By $(4.2 .2), \bar{g}=0$, and so $\bar{K}$ is trivial. In particular, $\bar{\Delta}=1$. The result now follows from (4.1.2) and (4.2.2).

For prime power periods, using the above proposition, we get the following sharpened version of Corollary 6 in [6].

Corollary 4.4. Suppose $q$ is a power of a prime $p$, and $\Delta \doteq 1(\bmod p)$. Then $q \leq g+1$.

To make these results more easily applicable to examples, it is useful to make a few observations regarding the relationship between the minimum number of crossings and the genus of a knot, which we will do in the remainder of this section.

Let $n$ be the minimum number of crossings of $K$, i.e., the least number among the number of crossings in all diagrams representing $K$. For a real number $x$, let $[x]$ denote the greatest integer $\leq x$. 
LEMMA 4.5. Suppose $K$ is not the $(n, 2)$-torus knot. Then $g \leq[n / 2]-1$.

Proof. Let $s$ be the number of the Seifert circles in an $n$ - crossing diagram for $K$. Then the Euler characteristic of the corresponding Seifert surface $F$, is $-n+s$, i.e., $1-2 g(F)=-n+s$, where $g(F)$ is the genus of the surface $F$. The nontriviality of the knot $K$, implies that $s \geq 2$. If $n$ is even, $s$ has to be odd, and so $s \geq 3$. On the other hand, if $n$ is odd, $s=2$ iff $K$ is the $(n, 2)$-torus knot, and so $s \geq 4$. As $g \leq g(F)$, the result follows.

The following two propositions follow from (4.2.1), 4.3 and 4.5.

Proposition 4.6. With $n$ as above, we have $q \leq n-1$. Moreover, if $n$ is odd and $K$ is not the $(n, 2)$ torus knot, then $q \leq n-2$.

Proposition 4.7. If $q$ is a power of a prime $p, K$ is not the $(n, 2)$-torus knot, and $[n / 2] \leq q$, then $\bar{g}=0$ and exactly one of the following possibilities holds.

(i) $q=g+1=[n / 2]$, and $\Delta \doteq 1$ (mod) $p$.

(ii) $q=g+1=[n / 2]$, $\operatorname{deg} \Delta=2(q-1)$, and $\Delta \doteq(1+t+$ $\left.t^{2}\right)^{q-1}(\bmod p)$.

(iii) $q=2 g+1, \operatorname{deg} \Delta=q-1$, and $\Delta \doteq(1+t)^{q-1}(\bmod p)$.

5. Applications to 10- and 11-Crossing Knots. The criteria for periodicity obtained in the previous sections can yield, when combined appropriately, an efficient procedure to determine the possible periods of knots. We illustrate it here in the case of 10- and 11crossing knots. Note that the periods of 10 -crossing knots have been determined before (see $[\mathbf{1}, \mathbf{3}, \mathbf{5}, \mathbf{1 3}, \mathbf{1 4}, \mathbf{1 6}, \mathbf{1 9}, \mathbf{2 0}, \mathbf{2 1}]$ ), whereas only a few specific examples of 11-crossing knots have been dealt with (see [16]). Here we obtain a unified proof for some of these known results for 10-crossing knots, and obtain new results in case of 11-crossing knots. In the discussion below, we follow the knot tables in [3] and [15] for the notation and information concerning the 10- and 11-crossing knots. 


\section{Proposition 5.1.}

(i) No 10-crossing knot has a period greater than 5 .

(ii) At most four 10-crossing knots can have period 5; these are: $10_{123}, 10_{124}, 10_{132}$, and $10_{137}$.

Proposition 5.2.

(i) The only possible periods for an 11-crossing knot are: 2, 3, 4, 5, and 11 .

(ii) There is exactly one 11-crossing knot of period 11, namely $11_{1}$.

(iii) At most three 11-crossing knots can have period 5; these are: $11_{224}, 11_{471}$, and $11_{473}$.

In order to prove the above assertions, suppose that $K$ is an $n$ crossing knot of period $q$, where $n \in\{10,11\}$. Let $\Delta, g, \bar{\Delta}$, and $\bar{g}$ be as in Section 4 . Note that the torus knot $11_{1}$ is periodic with periods 2 and 11 and has no other periods (see Corollary 6 of [13]). Hereafter, assume that $K \neq 11_{1}$. We first observe the following.

\section{LEMMA 5.3.}

(i) $g \leq 4$.

(ii) $q \leq 9$.

(iii) $q \notin\{6,8\}$.

(iv) If $q=9$, then $g=4, \bar{\Delta}=1, \operatorname{deg} \Delta=8$, and $\Delta \doteq(1+$ $t)^{8}(\bmod 3)$.

(v) If $q=7$, then $g=3, \bar{\Delta}=1, \operatorname{deg} \Delta=6$, and $\Delta \doteq(1+$ $t)^{6}(\bmod 7)$.

(vi) If $q=5$, then $\bar{\Delta}=1$ and exactly one of the following holds:

(a) $g=4$ and $\Delta \doteq 1(\bmod 5)$.

(b) $g=4$, deg $\Delta=8$, and $\Delta \doteq\left(1+t+t^{2}\right)^{4}(\bmod 5)$.

(c) $g=2, \operatorname{deg} \Delta=4$, and $\Delta \doteq(1+t)^{4}(\bmod 5)$.

Proof. Assertions (i) and (ii) follow from 4.5 and 4.6 respectively, whereas (iii) follows from (i) and (4.2.2). The rest follows directly from 4.7.

In view of the above observations, it is clear that the only periods possible for $K$ are $2,3,4,5,7$, and 9 . We now deal separately with the 10- and 11-crossing knots. 
First, observe that out of the total 165 knots with 10 crossings, only 26 have a degree 8 Alexander polynomial, none of which satisfies the congruence in (iv) of 5.3. Hence no 10-crossing knot has period 9. Now, there are 93 10-crossing knots with a degree 6 Alexander polynomial and only one of them satisfies the congruence in $(\mathrm{v})$ of 5.3. So just one 10-crossing knot, viz., $10_{105}$, may have period 7 . Furthermore, for $\Delta=\Delta_{10_{105}}=t^{6}-8 t^{5}+22 t^{4}-29 t^{3}+22 t^{2}-8 t+1$, we have, $\Delta(-1)=13 \times 7$, and if $10_{105}$ had period $7, \bar{\Delta}$ would have to be 1 ; now, since $f_{7}(13)>1$, we find that 2.8 is contradicted. Consequently, $10_{105}$ cannot have period 7 . Next, consider the congruences and degree conditions in (vi) of 5.3. We see that just 2 of the 26 degree 8 polynomials (viz., those of $10_{123}$ and $10_{124}$ ) satisfy (b), only 4 out of the 44 degree 4 polynomials (viz., those of $10_{18}, 10_{24}, 10_{132}$ and $10_{137}$ ) satisfy $(\mathrm{c}$ ), and finally, only one polynomial (viz., that of $10_{55}$ ) is trivial modulo 5 . Moreover, for the knots $10_{18}$ and $10_{24}$, we have $\Delta=4 t^{4}-14 t^{3}+19 t^{2}-14 t+4$, so $\Delta(2)=4$, and for $10_{55}$, we have $\Delta=5 t^{4}-15 t^{3}+21 t^{2}-15 t+5$, so $\Delta(2)=19$. Since $f_{5}(2)>2$ and $f_{5}(19)>1$, applying $(2.8)$ we see that $10_{18}, 10_{24}$, and $10_{55}$ do not have period 5. This completes the proof of (5.1).

Applying (5.3) to the 552 knots with 11 crossings, we see that there are at most 2 of period 9 (viz., $11_{432}, 11_{528}$ ), 1 of period 7 (viz., $11_{519}$ ), and 13 of period 5 (viz., $11_{2}, 11_{5}, 11_{42}, 11_{55}, 11_{89}$, $11_{105}, 11_{176}, 11_{181}, 11_{224}, 11_{401}, 11_{471}, 11_{473}$ and $\left.11_{486}\right)$. Any periodic knot with period 9, also has period 3 . In the example following Theorem 2.8 we showed that $11_{432}$ does not have period 3 . Also $11_{528}$ has an irreducible Alexander polynomial, which does not satisfy the Murasugi conditions 4.1 for period 3. Thus both $11_{432}$ and $11_{528}$ cannot have period 9 . Now we check, as in the above paragraph, if the remaining 15 knots satisfy the criterion in 2.8,(i)). By looking at the prime factors of the polynomial evaluated at -1 , we find that $11_{519}$ cannot have period 7 , and only $11_{224}, 11_{471}, 11_{473}$ can possibly have period 5 . It may be noted that $11_{471}$ and $11_{473}$ have the trivial Alexander polynomial. This completes the proof of 5.2 .

REMARK 5.4. It may be noted that, in [1], the knots $10_{132}$ and $10_{137}$ are shown not to have period 5 , which we have been unable to show. Also using the skein polynomial results of [20], it can be seen that $11_{224}, 11_{471}$, and $11_{473}$ do not have period 5 . In the notation 
of [20], $P_{0}\left(11_{224}\right)=3+4 l^{2}+2 l^{4}$, and $P_{0}\left(11_{471}\right)=P_{0}\left(11_{473}\right)=$ $2 l^{-4}+6 l^{-2}+7+2 l^{2}$.

REMARK 5.5. Weeks' program (see [1] ) computes the isometry group for a hyperbolic knot complement if the canonical ideal cell division is a triangulation. This gives an alternative way of finding periods of knots which satisfy this condition. In [1], the orders of these isometry groups are listed for links with less than 11 crossings. Clearly this could be done for 11-crossing knots as well; however, when the cell division is not a triangulation, Weeks' program fails to give any information regarding periodicity. The knot $10_{123}$ is an example where the program does not compute the symmetries, but using the Alexander polynomial and genus results, we can show that the only periods this knot can have are 2, 4 and 5 . It is easy to see that, in fact, it does have period 5. By 5.1 it follows that it does not have a period greater than 5 . Period 3 can be ruled out by using the fact that it has genus 4 , and then applying 3.2 and (4.1.2).

\section{REFERENCES}

[1] C. Adams, M. Hildebrand and J. Weeks, Hyperbolic invariants of knots and links, Trans. Amer. Math. Soc., 326 (1991), 1-56.

[2] G. Bredon, Introduction to Compact Transformation Groups, Academic Press, New York, 1972.

[3] G. Burde and H. Zieschang, Knots, Studies in Math. 5, de Gruyter, Berlin, 1985.

[4] V. Chumillas and J. M. Montesinos, The homology of cyclic and irregular dihedral coverings branched over homology spheres, Math. Ann., 280 (1988), 483-500.

[5] J. F. Davis and C. Livingston, Alexander polynomials of periodic knots, Topology, 30 (1991), 551-564.

[6] A. Edmonds, Least area Seifert surfaces and periodic knots, Topology and its Appl., 18 (1984), 109-113.

[7] E. Flapan, Infinitely periodic knots, Canad. J. Math., 37 (1985), 17-28.

[8] R. Fox, Knots and periodic transformations, in: Topology of 3-manifolds (ed. M. K. Fort), Prentice-Hall, Englewood Cliffs, N.J., (1962), 177-182.

[9] C. McA. Gordon, Some Aspects of Classical Knot Theory, in Knot Theory (ed. J. C. Hausmann), Lecture Notes in Math. 685, Springer-Verlag, Berlin, (1978), 1-60.

[10] I. Kaplansky, Modules over Dedekind rings and valuation rings, Trans. Amer. Math. Soc., 72 (1952), 327-340. 
[11] C. Livingston, Classical knot theory, Mathematical Manographs, MAA Washington D.C., 1993.

[12] D. A. Marcus, Number Fields, Springer-Verlag, New York, 1977.

[13] K. Murasugi, On periodic knots, Comment. Math. Helv., 46 (1971), 162-174.

[14] K. Murasugi, Jones polynomials of periodic links, Pacific J. Math., 131 (1988), 319-329.

[15] K. Perko, Invariants of 11-crossing knots, Publ. Math. d'Orsay (1980).

[16] J. Przytycki, On Murasugi's and Traczyk's criteria for periodic links, Math. Ann., 283 (1989), 465-478.

[17] H. Seifert, Uber das Geschlecht von Knoten, Math. Ann., 110 (1934), 571-592.

[18] P. Traczyk, A Criterion for knots of period 3, (1988), preprint.

[19] P. Traczyk, $10_{101}$ has no period 7 : A Criterion for Periodicity of Links, Proc. Amer. Math. Soc., 108 (1990), 845-846.

[20] P. Traczyk, Periodic knots and the skein polynomial, Invent. Math., 106 (1991), 73-85.

[21] Y. Yokota, The Jones polynomial of periodic knots, Proc. Amer. Math. Soc., 113 (1991), 889-894.

Received August 13, 1992, revised May 5, 1993 and accepted for publication June 14, 1993.

UNIVERSITY OF NEVADA

RENO, NV 89557

E-mail address: naik@math.unr.edu 




\section{PACIFIC JOURNAL OF MATHEMATICS}

Volume $166 \quad$ No. $2 \quad$ December 1994

Geometric aspects of Bäcklund transformations of Weingarten

submanifolds

STEVEN BUYSKE

Multipliers between invariant subspaces of the backward shift

225

ROBERT BRUCE CROFOOT

The Cauchy integral, analytic capacity and subsets of quasicircles

XIANG FANG

The number of lattice points within a contour and visible from the origin 295

Douglas Austin Hensley

On flatness of the Coxeter graph $E_{8}$

305

MASAKI IZUMI

Immersions up to joint-bordism

GUI SONG LI

Generalization of the Hilbert metric to the space of positive definite matrices

CARlangelo Liverani and Maciej WojtKowski

Periodicity, genera and Alexander polynomials of knots

SWATEE NAIK

On divisors of sums of integers. $\mathrm{V}$

ANDRÁS SÁRKÖZY and CAMERON LEIGH STEWART

Approximately inner automorphisms on inclusions of type $\mathrm{III}_{\lambda}$-factors

CARL WINSLøW

Correction to: "A convexity theorem for semisimple symmetric spaces"

KARL-HERMANN NEEB

Correction to: "Periodic points on nilmanifolds and solvmanifolds"

EDWARD KEPPELMANN

Correction to: "Partially measurable sets in measure spaces" 